\title{
Technological Approaches to Thermomechanical Processing of Quartz Glass Parts
}

\author{
Anna Basharova ${ }^{1,}{ }^{*}$, Aleksey Schenyatskiy ${ }^{1}$, and Mikhail Kotelnikov ${ }^{1}$ \\ ${ }^{1}$ Kalashnikov Izhevsk State Technical University, Izhevsk, Russian Federation, 426069
}

\begin{abstract}
The article discusses technological approaches applied to the processing of products made of quartz glass and used as components of gyroscopes and navigation devices. The developed block diagram of technological processes gives an idea of the life cycle of manufacturing a sensitive element - a gyroscope resonator, as well as input and output parameters at each of the manufacturing processes. Simulation of thermomechanical processing of samples in CAE Ansys with specified parameters of surface roughness gives an idea of the course of the process and technological modes. The selected temperature regimes make it possible to design a thermomechanical treatment process in order to carry out a practical study of the process.
\end{abstract}

\section{Technological approaches to the processing of quartz glass}

In modern production, it is relevant to solve problems of the selection of technological methods and approaches to the manufacture of parts and blocks of navigation devices. A sensitive element of the gyroscope - the resonator is made of quartz glass and has a small size and complex geometric profile. The shaping and further processing of the resonator is represented by complex processes such as diamond grinding, thermomechanical processing, chemical etching and polishing. Since technical requirements for the surface and shape are high, the problem of approaches and methods of processing a part becomes urgent.

The aim of the work is to develop a technological approach to interoperative processing of a part made of quartz glass based on an analysis of the processes occurring during grinding of a part with a diamond wheel having different grain sizes and concentration of diamonds in the wheel, which affect the purpose of processing modes and the quality of the resulting surface. The approach to thermomechanical processing of parts made of quartz glass is a gradual heating of the environment to the temperature when the surface roughness of the part changes, but does not lead to a change in the accuracy of the part's geometry.

\footnotetext{
* Corresponding author: phoenix-anya@mail.ru
} 


\subsection{Block diagram of processing of quartz glass parts}

Figure 1 shows a block diagram of the processing of parts made of quartz glass, taking into account such types of processing as diamond grinding [1,2] - shaping a part from a quartz glass blank, thermomechanical processing - smoothing the surface of the part and removing residual stresses $[3,4]$, chemical etching in hydrofluoric acid - etching off the outer (unalloyed) layers $[5,6]$.

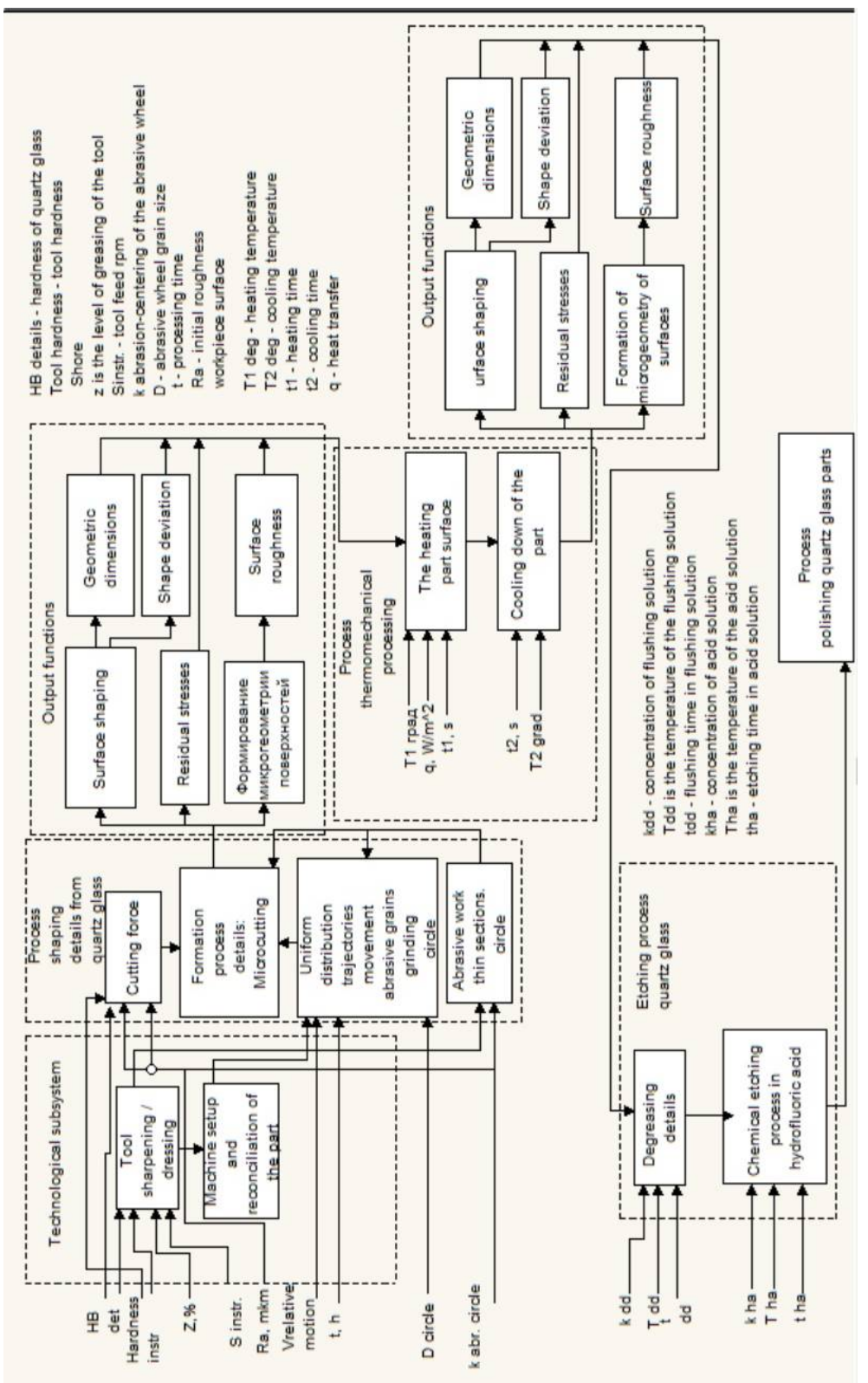

Fig. 1. Block diagram of diamond grinding processes, thermomechanical processing and chemical etching of quartz glass parts. 
The structural diagram is represented by a set of individual subsystems that determine the functioning of treatment process with displaying input and output parameters, as well as disturbing factors; it shows the mechanisms of surface shaping and formation of microgeometry of surfaces based on their mathematical description, it seems possible to purposefully control the treatment process. The group of processes of Technological Subsystem, as well as the process of shaping a quartz glass part, is aimed at solving the main problem - shaping the surface of the part by grinding with a bonded abrasive with diamond wheels. The thermomechanical treatment process is functionally used for shortterm melting of the surface layers of the workpiece to change the roughness parameter, while eliminating the deepest scratches from the abrasive. The etching process of quartz glass is an auxiliary cycle in the treatment, but it displays a qualitative picture of the result of abrasive and thermomechanical treatments, which leads to timely correction of the input and disturbing factors of previous processes with previously identified defects. The polishing process is discussed in detail in article [7].

\section{Simulation of thermomechanical treatment process}

Simulation was carried out in CAE Ansys, a $4 \times 4 \times 6 \mathrm{~mm}$ sample was used with the specified characteristics of quartz glass presented in Table 1.

Table 1. Quartz glass characteristics

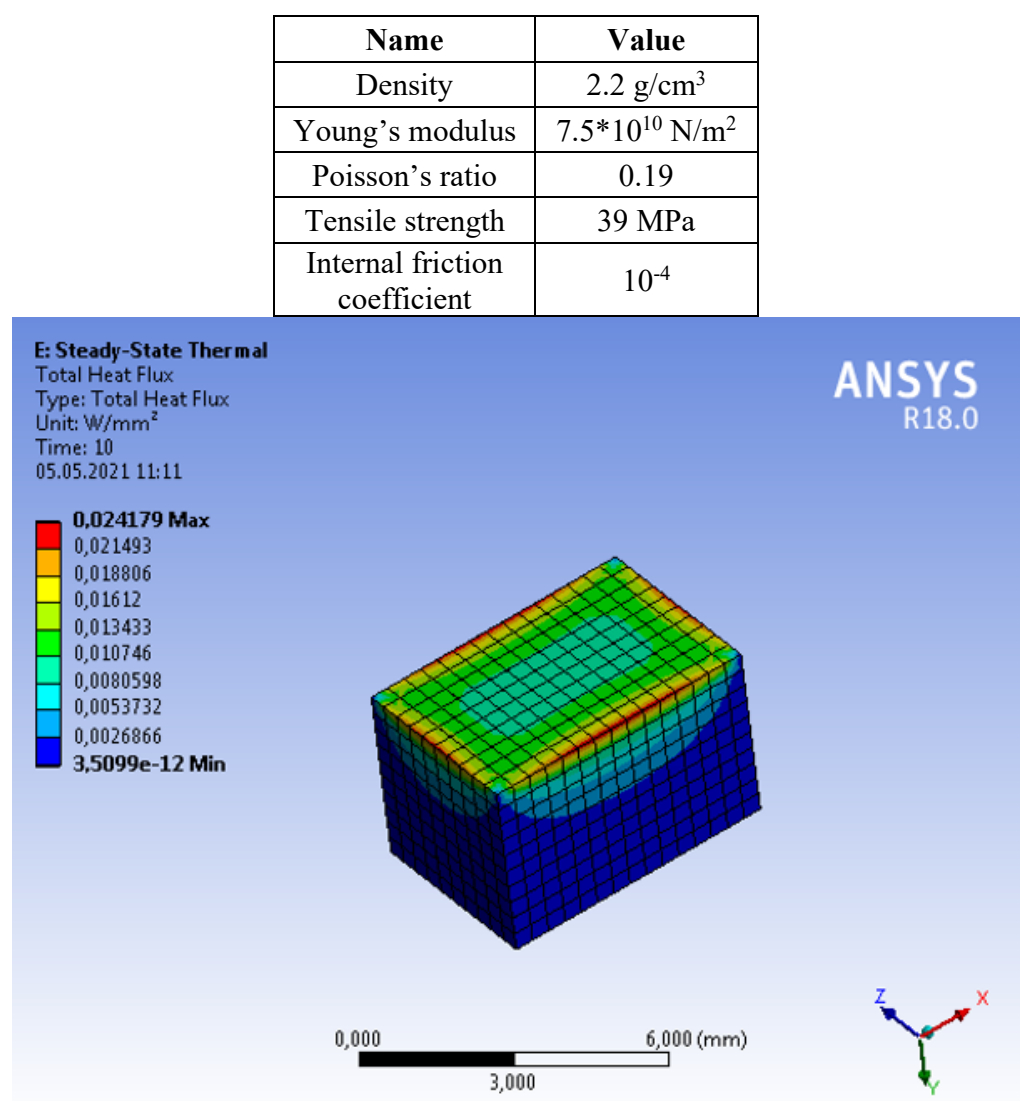

Fig. 2. The result of simulation of a quartz glass sample with a size of $4 \times 4 \times 6 \mathrm{~mm}$ 
As can be seen, the highest value of the heat flux is equal to $0.024179 \mathrm{~V} / \mathrm{mm}^{2}$ occurs in the edge zones of heating the sample, which is most important for real samples of parts used as a gyroscope resonator (Fig. 4) [8], where most often the appearance of defects occurs in the responsible part. Taking into account the division of the mesh into finite elements, it can be concluded that the maximum heating depth is $1.6 \mathrm{~mm}$ per $1 / 4$ of the sample size.

Next, a sample with dimensions of $1 \times 1 \times 1 \mathrm{~mm}$ was taken with an imitation of surface roughness after processing by grinding with a diamond wheel with a grain size of $125 / 100$ on a given segment, shown in Figure 3.

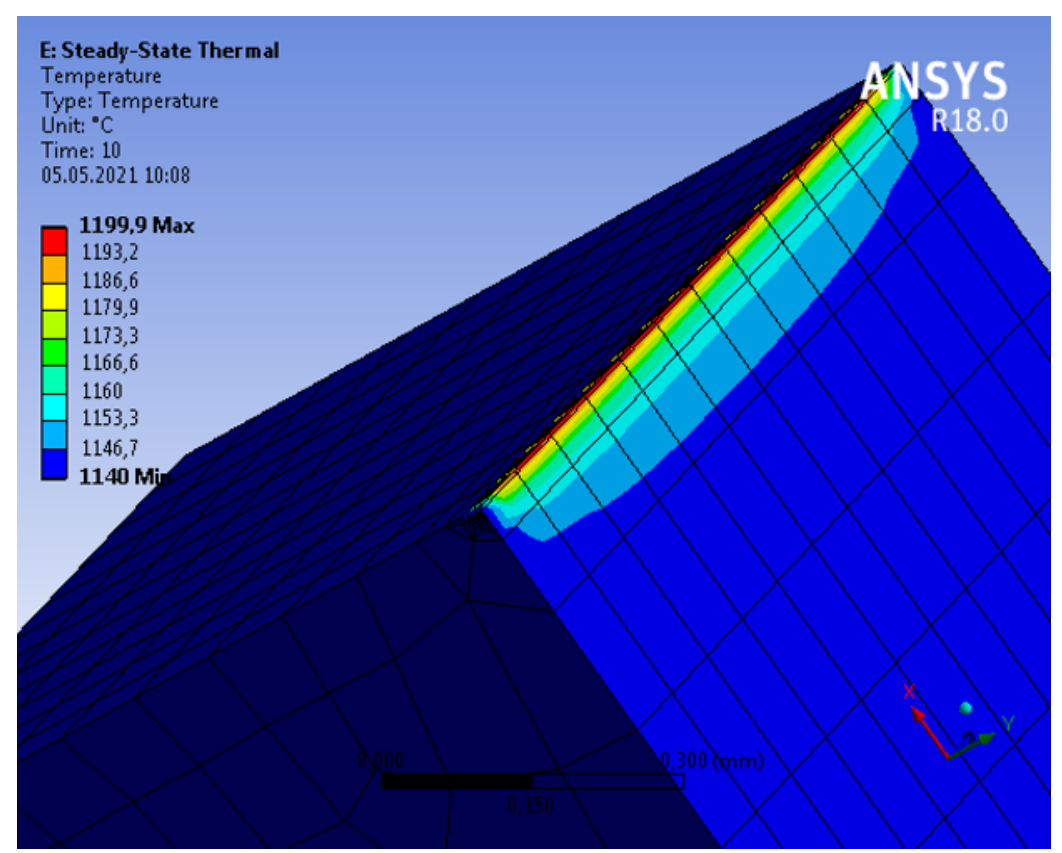

Fig. 3. The result of sample simulation with surface roughness imitation after diamond wheel processing.

With a given heat flux of $0.02 \mathrm{~V} / \mathrm{mm}^{2}$, the depth of heating to a temperature of $1140{ }^{\circ} \mathrm{C}$ is $1 / 5$ of the sample size.

The maximum temperature in the zone of surface roughness imitation after grinding wheel processing with a grain size of $125 / 100$ is $1199.9^{\circ} \mathrm{C}$, which is closest to the melting point of quartz glass.

For a real product (Fig. 4) with small geometric dimensions and thickness, the issue of uniformity and heating depth will be as relevant as for the sample under consideration. 


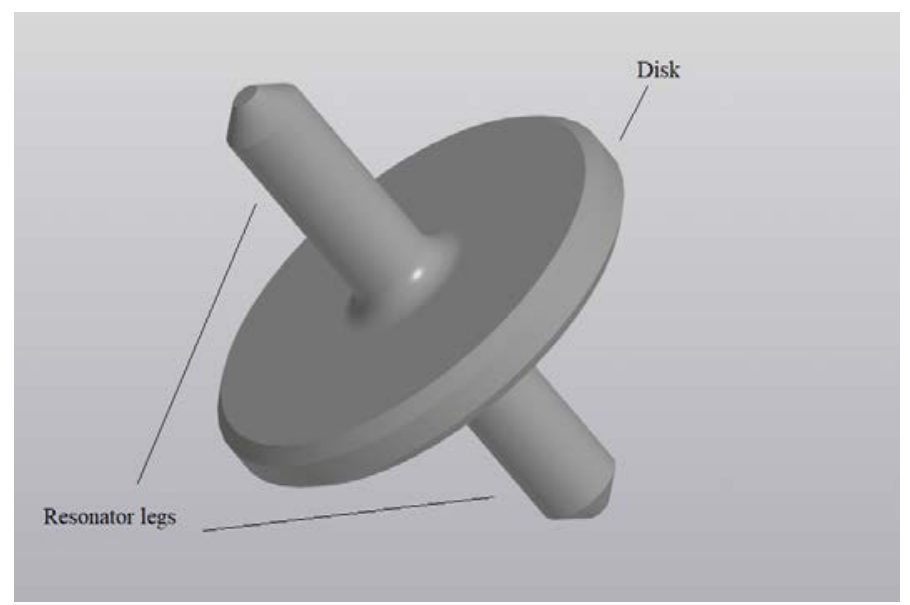

Fig. 4. Volumetric model of a rod sensitive element

\section{Conclusion}

Thus, having simulated the thermomechanical treatment process of a quartz glass sample, one can conclude that when performing a step-by-step approach to processing a part - diamond grinding - thermomechanical treatment - chemical etching reduce the risk of deep defects from diamond processing and can increase the figure of merit of the finished product, which must be confirmed by practical research results.

When developing technological processes for the combined processing of parts of small dimensions and thicknesses, it is necessary to take into account the heating amount and the heat flow direction to improve the quality of the resulting surface of the part.

\section{References}

1. Yu.K. Novoselov, S.M. Bratan. Modeling the processes of interaction between the grinding wheel and the workpiece during finishing grinding. Bulletin of SevNTU: collection of scientific papers. Issue 118/2011. Series: Mechanical engeneering and transport. - Sevastopol, 2011. pp. 92-106.

2. A.G. Kolesov. Improving the efficiency of processing precision surfaces of elastic plates in the operations of grinding and finishing: dissertation abstract of Cand. tech. Sciences. - Sevastopol, 2017. - 20 p.

3. B.S. Lunin, S.N. Torbin. Formation of surface defects of quartz glass during heat treatment. Moscow University Bulletin. Ser. 2. Chemistry. 2005. Vol. 46. No. 6.

4. B.S. Lunin, S.N. Torbin. Internal friction in quartz glass at moderate temperatures. Moscow University Bulletin. Ser. 2 Chemistry. 2000. Vol. 41. No. 2.

5. A.N. Trufanov, I.G. Naymushin. On the model of thermomechanical behavior of quartz glass and structures made of them. PNRPU Bulletin. Mechanics No. 3 pp. 85-99 (2010).

6. E.A. Akhmetshin, A.G. Cherednichenko. Investigation of quartz decoration processes by chemical etching method. Advances in chemistry and chemical technology. Volume XXXI. 2017 No. 15. pp. 66-69. 
7. A.A. Basharova, M.A. Kotelnikov, A.V. Shchenyatskiy. Technological approaches to processing of quartz glass parts. Bulletin of modern technologies. No. 4 (20) - 2020. pp.19-23.

8. A.V. Shchenyatskiy, M.A. Kotelnikov, A.A. Basharova. Influence of design parameters of the sensing element on technical characteristics of the solid-state wave gyroscope. Bulletin of PNRPU. No. 2 - 2017 (Vol.19), pp. 92-105. 